\title{
Achievable Rate Analysis in Network-Coded Cooperative Communications with Multiple Relay Nodes
}

\author{
Sushant Sharma $^{\star} \quad$ Yi Shi $^{\star} \quad$ Y. Thomas Hou ${ }^{\star}$ Sastry Kompella ${ }^{\dagger}$ \\ * Virginia Polytechnic Institute and State University, Blacksburg, VA, USA \\ $\dagger$ U.S. Naval Research Laboratory, Washington D.C., USA
}

\begin{abstract}
Network-coded cooperative communications (NC$\mathrm{CC})$ refers to the use of network coding (NC) in cooperative communications (CC). Prior studies have shown that NC has the potential to improve the performance of $\mathrm{CC}$ when there are multiple sessions in the wireless network. These studies were done for the case when multiple sessions are sharing a single relay node. However, how NC-CC behaves when multiple relay nodes are employed remains an open problem. In this paper, we explore this problem by analyzing the achievable rate of each session in this setting. We develop closed form formulas for the mutual information and the achievable data rate for each session and show that prior results for a single relay is a special case of our result. Our findings in this paper offer an important building block on the theory of NC-CC.
\end{abstract}

\section{INTRODUCTION}

Cooperative communications (CC) is an important technique to improve the performance of a wireless network [5]. Unlike MIMO, which requires the use of multiple antennas at each node, CC only employs a single antenna at each node and exploits diversity by cooperating with antennas on other nodes in the network. There has been extensive research at the physical layer that exploits cooperations among distributed antennas [1], [3], [6].

Recently, it was found that network coding (NC) can further improve the performance of CC by combining data streams at a relay node [2], [9], [10], [13], [14]. This application of NC in $\mathrm{CC}$ is called network-coded CC (or NC-CC). NC has been shown to improve the outage probability ([2], [9]), packet error rates $([13])$, and data rates ([10], [14]) for CC. These studies were done for the case when multiple sessions are sharing a single relay node. However, how NC-CC behaves when multiple relay nodes are employed remains an open problem.

In this paper, we study $\mathrm{NC}-\mathrm{CC}$ when there are multiple relay nodes. Our goal is to develop closed form formulas for the mutual information and the achievable data rate for each session. We consider the case of analog network coding (ANC) [4] and amplify-and-forward (AF) CC [7] at each relay node. Through an in-depth analysis, we derive the mathematical equations for mutual information and achievable data rate for each session and show that prior results for a single relay is a special case of our result. Our findings in this paper offer an important building block on the theory of NCCC. To demonstrate the application of our theoretical result, we apply it in a numerical study to understand the impact on a session's achievable rate when different sets of relay nodes are employed.

The remainder of this paper is organized as follows. In Section II, we review state-of-the-art result on NC-CC when

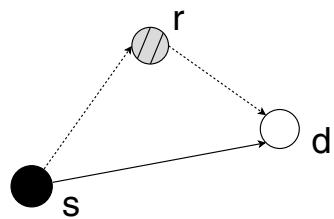

Fig. 1. A three-node relay channel for CC.

only a single relay node is employed. In Section III, we study NC-CC with multiple relay nodes and develop formulas for the mutual information and achievable data rate of each session. Section IV illustrates our results in a numerical study. Section V concludes this paper. Table I lists all notation used in this paper.

\section{BACKGROUND}

As a simple example, Fig. 1 shows a three-node relay channel for $\mathrm{CC}$. In this example, source node $s$ intends to transmit to destination node $d$ and will exploit relay node $r$ for possible performance improvement. Assume the time frame for transmission is divided into two time slots. In the first time slot, $s$ transmits to $d$, and is overheard by the relay node $r$. Relay node $r$ then amplifies the received signal, and then retransmits the amplified signal in the second time slot. The destination node $d$ can now combine the two copies of the same signal coming from two different paths. This cooperative relay channel in Fig. 1 can be treated as a single-input twooutput complex Gaussian channel [7] and the achievable data rate between $s$ and $d$ in this channel is given as follows:

$$
\begin{aligned}
& C_{\mathrm{CC}}(s, r, d)= \\
& \quad \frac{W}{2} \log _{2}\left(1+\mathrm{SNR}_{s d}+\frac{\mathrm{SNR}_{s r} \cdot \mathrm{SNR}_{r d}}{1+\mathrm{SNR}_{s r}+\mathrm{SNR}_{r d}}\right),
\end{aligned}
$$

where $\mathrm{SNR}_{u v}$ is the signal-to-noise ratio at the receiver $v$ when node $u$ transmits, and is given by $\mathrm{SNR}_{u v}=\frac{\left|h_{u v}\right|^{2} P_{u}}{\sigma^{2}}, \sigma_{v}^{2}$ is the variance of background noise at node $v, h_{u v}$ is the gain of the channel from node $u$ to node $v, P_{u}$ is the power at which node $u$ transmits signals, and $W$ is the channel bandwidth.

As for comparison, when $\mathrm{CC}$ is not used, i.e., $s$ transmits to $d$ without using $r$ (so-called direct transmission), the achievable rate from $s$ to $d$ is given as:

$$
C_{\mathrm{D}}(s, \emptyset, d)=W \log _{2}\left(1+\mathrm{SNR}_{s d}\right),
$$

where $\emptyset$ denotes that no relay node is used. It has been shown in [7] and [11] that $\mathrm{CC}$ has the potential to increase achievable 
TABLE I

\section{NOTATION}

\begin{tabular}{|c|l|}
\hline Symbol & Definition \\
\hline$\alpha_{r}$ & Amplification factor at relay node $r$ \\
$\sigma_{v}^{2}$ & Variance of background noise at node $v$ \\
$\sigma_{z_{v}^{\text {ANC }}}^{2}$ & Variance of ANC noise at node $v$ \\
$h_{u v}$ & Effect of path-loss, shadowing, and fading \\
$z_{v}$ & from node $u$ to node $v$ \\
$z_{v}^{\mathrm{ANC}}$ & White Gaussian background noise at node $v$ \\
ANC noise at node $v$ \\
$C_{\mathrm{NC}-\mathrm{CC}}\left(s_{i}, \mathcal{S}, \mathcal{R}, d_{i}\right)$ & Achievable rate for session $\left(s_{i}, d_{i}\right): s_{i} \in \mathcal{S}$ \\
$I_{\mathrm{NC}-\mathrm{CC}}\left(s_{i}, \mathcal{S}, \mathcal{R}, d_{i}\right)$ & employs NC-CC using the set of relays $\mathcal{R}$ \\
& Mutual information for session $\left(s_{i}, d_{i}\right):$ \\
$P_{u}$ & $s_{i} \in \mathcal{S}$ that employs relay group $\mathcal{R}$ \\
$\mathcal{R}$ & Transmission power at node $u$ \\
$\mathcal{S}$ & The set of all relay nodes in the network \\
$\mathrm{SNR}_{u v}$ & The set of all source nodes in the network \\
$W$ & The signal to noise ratio between nodes $u$ and $v$ \\
& Total bandwidth available in the network \\
\hline
\end{tabular}

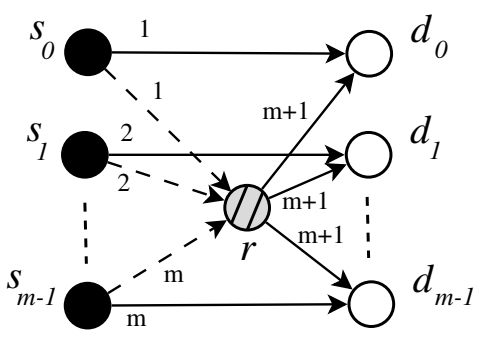

(a) $m$ source nodes sharing one relay node.

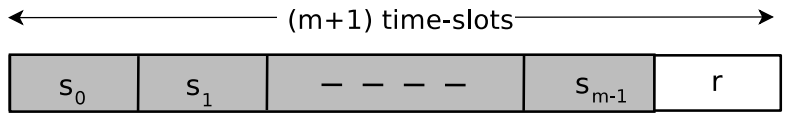

(b) Structure of a time frame.

Fig. 2. NC-CC with a single relay node.

rate over direct transmission, depending on the location of relay node and its channel statistics.

When there are $m$ source-destination sessions sharing a single relay node (as shown in Fig. 2), one can employ NC to combine the signals from the $m$ sources at the relay node and then forward the combined signal to all the destination nodes, i.e., NC-CC. Here, a time frame is divided into $(m+1)$ time slots (see Fig. 2(b)), with each of the first $m$ time slots being used for transmission by one of the source nodes. Again, each of such transmissions is received by its corresponding destination node and overheard by the relay node. The relay node will then apply $\mathrm{NC}$ to combine all the $m$ received analog signals. The combined signal is then amplified and forwarded by the relay node to all destination nodes in the $(m+1)$-th time slot. The achievable rate for a session under NC-CC is given by [10]:

$$
\begin{gathered}
C_{\mathrm{NC}-\mathrm{CC}}\left(s_{i}, \mathcal{S}, r, d_{i},\right)=\frac{W}{m+1} \log _{2}\left(1+\mathrm{SNR}_{s_{i} d_{i}}\right. \\
\left.+\frac{\mathrm{SNR}_{s_{i} r} \mathrm{SNR}_{r d_{i}}}{\left|\mathcal{S}_{r}\right| \frac{\sigma_{z_{d_{i}}^{\mathrm{ANC}}}^{2}}{\sigma_{d_{i}}^{2}}+\mathrm{SNR}_{r d_{i}}+\frac{\sigma_{z_{d_{i}}}^{2}}{\sigma_{d_{i}}^{2}} \sum_{s_{j} \in \mathcal{S}} \mathrm{SNR}_{s_{j} r}}\right)
\end{gathered}
$$

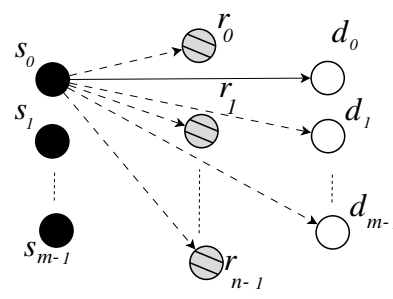

(a) Time slot 1 .

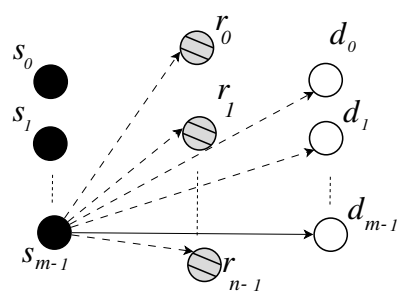

(c) Time slot $m$.

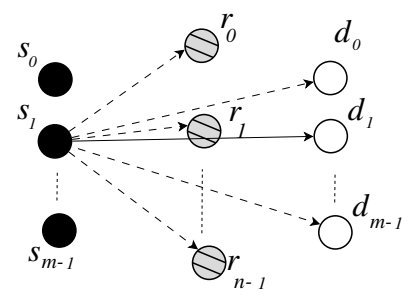

(b) Time slot 2 .

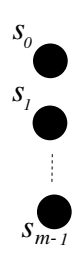

(d) Time slot $m+1$.

\begin{tabular}{|c|c|c|c|c|c|}
\hline $\mathrm{s}_{0}$ & $\mathrm{~s}_{1}$ & $\cdots \cdots \cdots$ & $\mathrm{s}_{\mathrm{m}-2}$ & $\mathrm{~s}_{\mathrm{m}-1}$ & $\mathcal{R}$ \\
\hline
\end{tabular}

(e) Time slot structure for NC-CC with multiple relay nodes.

Fig. 3. Data transfer under multi-relay NC-CC in $m+1$ time-slots.

where $\mathcal{S}=\left\{s_{0}, s_{1}, \cdots, s_{m-1}\right\}$ is the set of all the source nodes, and $\sigma_{z_{d_{i}}^{\mathrm{ANC}}}^{2}$ is the noise at destination $d_{i}$ due to ANC, and is given by

$$
\begin{array}{r}
\sigma_{z_{d_{i}}^{\text {ANC }}}^{2}=\sigma_{d_{i}}^{2}+(|\mathcal{S}|-1)\left(\alpha_{r} h_{r d_{i}}\right)^{2} \sigma_{r}^{2}+ \\
\sigma_{d_{i}}^{2} \sum_{s_{j} \in \mathcal{S}}^{s_{j} \neq s_{i}}\left(\frac{\alpha_{r} h_{s_{j} r} h_{r d_{i}}}{h_{s_{j} d_{i}}}\right)^{2},
\end{array}
$$

where $\alpha_{r}$ is the amplification factor for AF CC at relay node $r$ and is given by

$$
\alpha_{r}^{2}=\frac{P_{r}}{|\mathcal{S}| \sigma_{r}^{2}+\sum_{s_{i} \in S} P_{s_{i}}\left|h_{s_{i} r}\right|^{2}} .
$$

\section{NC-CC With Multiple Relay Nodes}

The NC-CC model in Fig. 2 employs only a single relay node. In this section, we study the general NC-CC model with multiple relay nodes, and derive mutual information and achievable data rate for each session.

We consider that there are $m$ sessions in the network (denoted as $\mathcal{S}=\left\{s_{0}, s_{1}, \cdots, s_{m-1}\right\}$ ) that are sharing $n$ relay nodes with NC-CC. This general multi-session multi-relay NC-CC model is illustrated in Fig. 3. In this figure, the source node of each session transmits in the first $m$ time slots, and each transmission is received by the destination nodes as well as the $n$ relay nodes. After the $m$ transmissions, all the relay nodes will apply $\mathrm{NC}$ to combine the signals received during the $m$ time slots. Figure 3(d) shows that the combined signal is amplified and simultaneously transmitted by all the relay nodes 
in the $(m+1)$-th time slot. The coherent signal reception at destination nodes can be facilitated by appropriate transmitter synchronization [8]. Figure 3(e) shows the time-slot structure for this general multi-session multi-relay model. Every session is allotted a time slot duration of $t$. Thus, the time slot duration available to each source node is $\frac{T}{m+1}=\frac{|\mathcal{S}| t}{|\mathcal{S}|+1}$. Therefore, the achievable rate for a session $\left(s_{i}, d_{i}\right)$ can be written as:

$$
\begin{aligned}
C_{\mathrm{NC}-\mathrm{CC}}\left(s_{i}, \mathcal{S}, \mathcal{R}, d_{i}\right)= & W \cdot \frac{\left(\frac{|\mathcal{S}| t}{|\mathcal{S}|+1}\right)}{|\mathcal{S}| t} I_{\mathrm{NC}-\mathrm{CC}}\left(s_{i}, \mathcal{S}, \mathcal{R}, d_{i}\right) \\
& =\frac{W}{|\mathcal{S}|+1} I_{\mathrm{NC}-\mathrm{CC}}\left(s_{i}, \mathcal{S}, \mathcal{R}, d_{i}\right)
\end{aligned}
$$

where $I_{\text {NC-CC }}\left(s_{i}, \mathcal{S}, \mathcal{R}, d_{i}\right)$ is the mutual information between the source $s_{i}$ and its destination node $d_{i}$.

Now we derive the mutual information $I_{\mathrm{NC} \text {-CC }}\left(s_{i}, \mathcal{S}, \mathcal{R}, d_{i}\right)$. First, the signal transmitted by source $s_{i}$ and received by destination $d_{i}$ can be written as:

$$
y_{s_{i} d_{i}}=h_{s_{i} d_{i}} x_{i}+z_{d_{i}} \text {. }
$$

Next, the signal transmitted by source $s_{i}$ and received by relay $r_{j}$ can be written as:

$$
y_{s_{i} r_{j}}=h_{s_{i} r_{j}} x_{i}+z_{r_{j}} \text {. }
$$

Finally, the simultaneous signals transmitted by all the relay nodes and received by destination node $d_{i}$ in the $(m+1)$-th time slot can be written as:

$$
\begin{aligned}
y_{\mathcal{S R} d_{i}} & =\sum_{r_{j} \in R}\left(\alpha_{r_{j}} h_{r_{j}} d_{i} \sum_{s_{k} \in \mathcal{S}} y_{s_{k} r_{j}}\right)+z_{d_{i}} \\
& =\sum_{r_{j} \in R}\left(\alpha_{r_{j}} h_{r_{j} d_{i}} \sum_{s_{k} \in \mathcal{S}}\left(h_{s_{k} r_{j}} x_{k}+z_{r_{j}}\right)\right)+z_{d_{i}},
\end{aligned}
$$

where the value of amplification factor at $r_{j}$ is:

$$
\alpha_{r_{j}}^{2}=\frac{P_{r_{j}}}{\sum_{s_{k} \in \mathcal{S}}\left(\sigma_{r_{j}+}^{2} P_{s_{k}}\left|h_{s_{k} r_{j}}\right|^{2}\right)} .
$$

A destination node $d_{i}$ has to extract signal for $x_{i}$ from the combined signal in (9). To extract the desired signal, a destination node $d_{i}$ can subtract the signals received from other source nodes in the initial $m$ time slots from this combined signal. The signals received by destination node $d_{i}$ from other source nodes during the first $m$ time slots can be written as:

$$
y_{s_{k} d_{i}}=h_{s_{k} d_{i}} x_{k}+z_{d_{i}}, \quad\left(s_{k} \in \mathcal{S}, s_{k} \neq s_{i}\right) .
$$

To remove the signal $x_{k}$ from the combined signal in (9), destination node $d_{i}$ can multiply the overheard signal for $x_{k}$ in (10) by $\frac{\sum_{r_{j} \in \mathcal{R}} \alpha_{r_{j}} h_{r_{j} d_{i}} h_{s_{k} r_{j}}}{h_{s_{k} d_{i}}}$, and then subtract this product from (9). When the signals for all $x_{k}$ 's are removed from (9), the copy of the desired signal extracted by $d_{i}$ can be written as:

$$
\begin{aligned}
y_{\mathcal{S R} d_{i}}= & x_{i} \sum_{r_{j} \in \mathcal{R}} h_{s_{i} r_{j}} \alpha_{r_{j}} h_{r_{j} d_{i}}+\sum_{s_{k} \in \mathcal{S}} \sum_{r_{j} \in \mathcal{R}} \alpha_{r_{j}} h_{r_{j} d_{i}} z_{r_{j}} \\
& +z_{d_{i}}-\sum_{s_{k} \in \mathcal{S}}^{s_{k} \neq s_{i}} \frac{\sum_{r_{j} \in \mathcal{R}} h_{s_{k} r_{j}} \alpha_{r_{j}} h_{r_{j} d_{i}}}{h_{s_{k} d_{i}}} \\
= & \sum_{r_{j} \in \mathcal{R}} \alpha_{r_{j}} h_{r_{j} d_{i}} y_{s_{i} r_{j}}+z_{d_{i}}+\sum_{s_{k} \in \mathcal{S}}^{s_{k} \neq s_{i}} \sum_{r_{j} \in \mathcal{R}} \alpha_{r_{j}} h_{r_{j} d_{i}} z_{r_{j}} \\
& -\sum_{s_{k} \in \mathcal{S}}^{s_{k} \neq s_{i}} \frac{\sum_{r_{j} \in \mathcal{R}} h_{s_{k} r_{j}} \alpha_{r_{j}} h_{r_{j} d_{i}}}{h_{s_{k} d_{i}}} z_{d_{i} .} .
\end{aligned}
$$

Due to the use of ANC and multiple relay nodes, we find in (11) that in addition to $z_{d_{i}}$, there are some new noise terms in the signal extracted by $d_{i}$. This new noise is called the ANC noise, and is written as:

$$
\begin{aligned}
z_{d_{i}}^{\mathrm{ANC}}= & z_{d_{i}}+\sum_{s_{k} \in \mathcal{S}}^{s_{k} \neq s_{i}} \sum_{r_{j} \in \mathcal{R}} \alpha_{r_{j}} h_{r_{j} d_{i}} z_{r_{j}} \\
& -\sum_{s_{k} \in \mathcal{S}}^{s_{k} \neq s_{i}} \frac{\sum_{r_{j} \in \mathcal{R}} h_{s_{k} r_{j}} \alpha_{r_{j}} h_{r_{j} d_{i}}}{h_{s_{k} d_{i}}} z_{d_{i}} .
\end{aligned}
$$

We can now write the variance of ANC noise as:

$$
\begin{aligned}
\sigma_{z_{d_{i}}^{\mathrm{ANC}}}^{2}=\sigma_{d_{i}}^{2}+ & (|\mathcal{S}|-1) \sum_{r_{j} \in \mathcal{R}}\left(\alpha_{r_{j}} h_{r_{j} d_{i}}\right)^{2} \sigma_{r_{j}}^{2} \\
& +\sum_{s_{k} \in \mathcal{S}}^{s_{k} \neq s_{i}} \sum_{r_{j} \in \mathcal{R}}\left(\frac{h_{s_{k} r_{j}} \alpha_{r_{j}} h_{r_{j} d_{i}}}{h_{s_{k}} d_{i}}\right)^{2} \sigma_{d_{i}}^{2} .
\end{aligned}
$$

From (12), we can make two important observations: (i) the variance in ANC noise increases monotonically as the group size of sessions sharing the same set of relays increase; and (ii) the variance in ANC noise increases monotonically with the size of the set of relays.

We can now write (7) and (11) in a compact matrix form as follows:

$$
\mathbf{Y}=\mathbf{H} x_{i}+\mathbf{B Z}
$$

where

$$
\begin{aligned}
& \mathbf{Y}=\left[\begin{array}{c}
y_{s_{i} d_{i}} \\
y_{\mathcal{S R} d_{i}}
\end{array}\right], \quad \mathbf{H}=\left[\begin{array}{c}
h_{s_{i} d_{i}} \\
\sum_{r_{j} \in \mathcal{R}} h_{s_{i} r_{j}} \alpha_{r_{j}} h_{r_{j} d_{i}}
\end{array}\right], \\
& \mathbf{B}=\left[\begin{array}{cccccc}
0 & 0 & \cdots & 0 & 1 & 0 \\
\alpha_{r} h_{r_{0} d_{i}} & \alpha_{r} h_{r_{1} d_{i}} & \cdots & \alpha_{r} h_{r_{n-1} d_{i}} & 0 & 1
\end{array}\right] \text {, and } \\
& \mathbf{Z}=\left[\begin{array}{c}
z_{r_{0}} \\
z_{r_{1}} \\
\cdot \\
\cdot \\
z_{r_{n-1}} \\
z_{d_{i}} \\
z_{d_{i}}^{\mathrm{ANC}}
\end{array}\right]
\end{aligned}
$$

We can model the above channel from $s_{i} \rightarrow d_{i}$ and from $s_{i} \rightarrow \mathcal{R} \rightarrow d_{i}$ as a one-input two-output complex Gaussian 
channel [7]. The mutual information between $s_{i}$ and $d_{i}$ can be written as:

$$
\begin{array}{r}
I_{\mathrm{NC}-\mathrm{CC}}\left(s_{i}, \mathcal{S}, \mathcal{R}, d_{i}\right)=\log \operatorname{det}\{\mathbf{I}+ \\
\left.\left(P_{s_{i}} \mathbf{H H}^{\dagger}\right)\left(\mathbf{B} E\left[\mathbf{Z} \mathbf{Z}^{\dagger}\right] \mathbf{B}^{\dagger}\right)^{-1}\right\},
\end{array}
$$

where

$$
E\left[\mathbf{Z Z}^{\dagger}\right]=\left[\begin{array}{cccccc}
\sigma_{r_{0}}^{2} & 0 & \cdots & 0 & 0 & 0 \\
0 & \sigma_{r_{1}}^{2} & \cdots & 0 & 0 & 0 \\
0 & 0 & \ddots & 0 & 0 & 0 \\
0 & 0 & \cdots & \sigma_{r_{n-1}}^{2} & 0 & 0 \\
0 & 0 & \cdots & 0 & \sigma_{d_{i}}^{2} & 0 \\
0 & 0 & \cdots & 0 & 0 & \sigma_{z_{d_{i}}^{\mathrm{ANC}}}^{2}
\end{array}\right] .
$$

Expanding (13) gives us the value of mutual information between $s_{i}$ and $d_{i}$ as follows:

$$
\begin{gathered}
I_{\mathrm{NC}-\mathrm{CC}}\left(s_{i}, \mathcal{S}, \mathcal{R}, d_{i}\right)=\log _{2}\left[1+\frac{P_{s_{i}}\left|h_{s_{i} d_{i}}\right|^{2}}{\sigma_{d_{i}}^{2}}+\right. \\
\left.\frac{P_{s_{i}}\left(\sum_{r_{j} \in \mathcal{R}} h_{s_{i} r_{j}} \alpha_{r_{j}} h_{r_{j} d_{i}}\right)^{2}}{\sigma_{z_{d_{i}}^{\mathrm{ANC}}}^{2}+\sum_{r_{j} \in \mathcal{R}} h_{r_{j} d_{i}}^{2} \alpha_{r_{j}}^{2} \sigma_{r_{j}}^{2}}\right],
\end{gathered}
$$

which can be rewritten as:

$$
\begin{aligned}
I_{\mathrm{NC}-\mathrm{CC}} & \left(s_{i}, \mathcal{S}, \mathcal{R}, d_{i}\right)=\log _{2}\left[1+\mathrm{SNR}_{s_{i} d_{i}}+\right. \\
& \left.\frac{\left(\sum_{r_{j} \in \mathcal{R}} \sqrt{\left.\frac{\mathrm{SNR}_{r_{j} d_{i}} \mathrm{SNR}_{s_{i} r_{j}}}{|\mathcal{S}|+\sum_{s_{k} \in \mathcal{S}} \mathrm{SNR}_{s_{k} r_{j}}}\right)^{2}}\right] .}{\frac{\mathrm{SNR}_{r_{j} d_{i}}}{\sigma_{d_{i}}^{2}}+\sum_{r_{j} \in \mathcal{R}}\left(\frac{\mathrm{SNC}_{d_{i}}^{2}}{|\mathcal{S}|+\sum_{s_{k}} \in \mathcal{S}} \mathrm{SNR}_{s_{k} r_{j}}\right.}\right)
\end{aligned}
$$

From (14), we can see that the value of mutual information depends directly on the individual relay nodes in $\mathcal{R}$ and the value of ANC noise. We can make two important observations by taking a close look at (14). First, a given session $\left(s_{i}, d_{i}\right)$ performing NC-CC with a group of relays $\left(r_{j}\right.$ 's) would prefer these relays to stay as close as possible to $s_{i}$. Intuitively, this is true because the signal that $r_{j}$ 's will receive from $s_{i}$ will have smaller noise component (or larger SNR) due to proximity to $s_{i}$. A mathematical explanation of this is that the term $\mathrm{SNR}_{s_{i} r_{j}}$ is in the denominator of (14). Second, $s_{i}$ would also prefer to have other $s_{k}$ 's in the session group to be far away from the relay nodes. This is because when the distance between the other source nodes (i.e., other $s_{k}$ 's) and the relay nodes increases, the value of channel gains between other $s_{k}$ 's and the relays become small, which will help reduce the ANC noise value at destination $d_{i}$. This can be observed in (12) where the value of ANC noise decreases monotonically with the decrease in the value of $h_{s_{k} r_{j}}$.

\section{NumericAl Results}

The goal of this section is to apply the achievable rate equations (6) and (14) in a numerical study to calculate the data rates of each session when different set of relay nodes are employed.

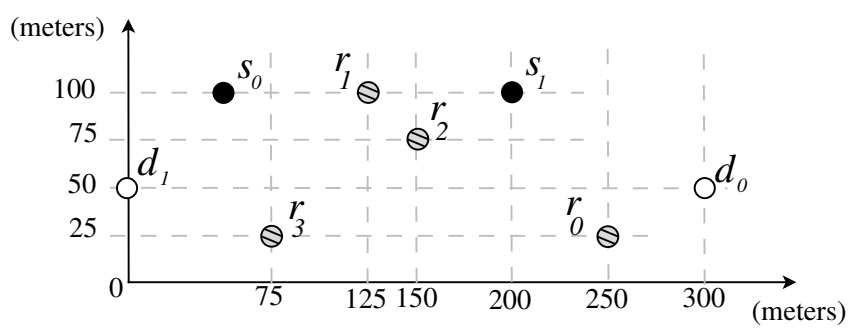

Fig. 4. Two-session four-relay network.

TABLE II

DATA RATES UNDER NC-CC WITH DIFFERENT SETS OF RELAY NODES.

\begin{tabular}{|c|c|c|c|}
\hline $\mathcal{R}$ & \multicolumn{2}{|c|}{ Data Rate (Mbps) } & Total \\
\cline { 2 - 3 } & $\left(s_{0}, d_{0}\right)$ & $\left(s_{1}, d_{1}\right)$ & $($ Mbps $)$ \\
\hline \hline $\mathcal{R}_{0}=\emptyset$ & 16.70 & 25.83 & 42.53 \\
$\mathcal{R}_{1}=\left\{r_{0}\right\}$ & 13.65 & 20.01 & 33.66 \\
$\mathcal{R}_{2}=\left\{r_{1}\right\}$ & 18.48 & 27.44 & 45.92 \\
$\mathcal{R}_{3}=\left\{r_{2}\right\}$ & 13.81 & 28.80 & 42.61 \\
$\mathcal{R}_{4}=\left\{r_{3}\right\}$ & 17.50 & 23.14 & 40.64 \\
$\mathcal{R}_{5}=\left\{r_{0}, r_{1}\right\}$ & 15.45 & 31.78 & 47.23 \\
$\mathcal{R}_{6}=\left\{r_{0}, r_{2}\right\}$ & 14.63 & 33.09 & 47.72 \\
$\mathcal{R}_{7}=\left\{r_{0}, r_{3}\right\}$ & 15.22 & 26.07 & 41.29 \\
$\mathcal{R}_{8}=\left\{r_{1}, r_{2}\right\}$ & 22.30 & 37.82 & 60.12 \\
$\mathcal{R}_{9}=\left\{r_{1}, r_{3}\right\}$ & 26.24 & 30.03 & 56.27 \\
$\mathcal{R}_{10}=\left\{r_{2}, r_{3}\right\}$ & 21.57 & 30.63 & 52.19 \\
$\mathcal{R}_{11}=\left\{r_{0}, r_{1}, r_{2}\right\}$ & 16.52 & $\mathbf{4 0 . 5 3}$ & 57.04 \\
$\mathcal{R}_{12}=\left\{r_{0}, r_{1}, r_{3}\right\}$ & 17.15 & 32.39 & 49.54 \\
$\mathcal{R}_{13}=\left\{r_{0}, r_{2}, r_{3}\right\}$ & 16.28 & 32.97 & 49.25 \\
$\mathcal{R}_{14}=\left\{r_{1}, r_{2}, r_{3}\right\}$ & $\mathbf{2 8 . 4 9}$ & 35.96 & 64.45 \\
$\mathcal{R}_{15}=\left\{r_{0}, r_{1}, r_{2}, r_{3}\right\}$ & 18.22 & 37.77 & 55.99 \\
\hline
\end{tabular}

\section{A. Simulation Settings}

Consider the network topology in Fig. 4 where there are two sessions $\left(s_{0}, d_{0}\right)$ and $\left(s_{1}, d_{1}\right)$. There are four relay nodes that can be employed, namely, $r_{0}, r_{1}, r_{2}$ and $r_{3}$. We assume that the channel bandwidth in the network is $W=20 \mathrm{MHz}$, the white Gaussian noise at all the nodes has a variance of $10^{-10} \mathrm{~W}$, and every node transmits at a power of $1 \mathrm{~W}$. We assume the channel gain between two nodes $u$ and $v$ is $\|u-v\|^{-4}$, where $\|u-v\|$ is the distance (in meters) between $u$ and $v$, the path loss index is 4 .

\section{B. Results}

We calculate the achievable rate of each session under NC$\mathrm{CC}$ when different set of relay nodes are used. Since there are four relay nodes, there are 16 possibilities (see first column in Table II), ranging from the trivial case of no relay node is used to the extreme case when all four relay nodes are used. Table II lists the data rates for each session under each of these 16 cases. When $\mathcal{R}=\emptyset$, Eq. (2) (for direct transmission) is used and the effective bandwidth available to each session is $\frac{W}{2}=10 \mathrm{MHz}$. The last column in Table II shows the sum of the data rates of both sessions.

For the 15 sets of relay nodes (excluding direct transmission), we observe that not every set is beneficial to both sessions, compared to the case of direct transmission. For session $\left(s_{0}, d_{0}\right)$, the sets of $\mathcal{R}_{1}, \mathcal{R}_{3}, \mathcal{R}_{5}, \mathcal{R}_{6}, \mathcal{R}_{7}, \mathcal{R}_{11}$, and $\mathcal{R}_{13}$ are not beneficial, whereas for session $\left(s_{1}, d_{1}\right)$, the sets of $\mathcal{R}_{1}$ and $\mathcal{R}_{4}$ are not beneficial. Some sets of relay nodes are beneficial to one session but not to the other session, e.g., 
relay groups $\mathcal{R}_{3}, \mathcal{R}_{5}, \mathcal{R}_{6}, \mathcal{R}_{7}, \mathcal{R}_{11}$, and $\mathcal{R}_{13}$ are beneficial to $\left(s_{1}, d_{1}\right)$ but not to $\left(s_{0}, d_{0}\right)$. Also, the most beneficial set of relay nodes for $\left(s_{0}, d_{0}\right)$ is $\mathcal{R}_{14}$, and the most beneficial set of relay nodes for $\left(s_{1}, d_{1}\right)$ is $\mathcal{R}_{11}$.

Furthermore, as per our discussion at the end of Section III, we can observe that $\mathcal{R}_{4}$ is beneficial to session $\left(s_{0}, d_{0}\right)$ but not to session $\left(s_{1}, d_{1}\right)$ due to its proximity to source $s_{0}$. Similarly, $\mathcal{R}_{3}$ is beneficial to $\left(s_{1}, d_{1}\right)$ but not to $\left(s_{0}, d_{0}\right)$ due to its proximity to $s_{1}$ compared to $s_{0}$. For some other relay groups, the conclusion may not be made simply by observing the location of individual nodes. This is where our derived equations can help in determining whether a session group should be matched with a relay group or not. It is important to realize that without deriving the mathematical equations for achievable data rate and mutual information, it is impossible to construct a table such as the one shown in Table II. The formulas we developed can thus help us to identify the optimal set of relay nodes for some particular objective (e.g., maximum sum rate or max-min). The design of such an algorithm, however, is beyond the scope of this paper.

\section{CONCLUSiON}

In this paper, we studied how NC-CC behaves when multiple relay nodes are employed. We derived closed form equations for mutual information and achievable data rate for each session under such setting. Our derivation was based on generalizing the three-node relay channel model with multiple relays and then taking consideration of multiple sessions. Our results in this paper offer an important building block on the theory of NC-CC. To demonstrate the application of our theoretical result, we applied it in a numerical study to understand the impact on a session's achievable rate when different sets of relay nodes are employed.

The results of this paper pave the way for the research community to explore new problems such as finding an optimal set of relay nodes for a given objective. This is our current research and its results will be reported in a future paper.

\section{ACKNOWLEDGMENTS}

The work of Y.T. Hou, S. Sharma, and Y. Shi was supported in part by the NSF under Grant CCF-0946273. The work of S. Kompella was supported in part by the ONR.

\section{REFERENCES}

[1] B. Aazhang, R.S. Blum, J.N. Laneman, K.J.R. Liu, W. Su, and A. Wittneben (editors), IEEE Journal on Selected Areas in Com- munications - Special Issue on Cooperative Communications and Networking, vol. 25, no. 2, Feb. 2007.

[2] X. Bao and J. Li, "Adaptive network coded cooperation (ANCC) for wireless relay networks: matching code-on-graph with network-on-graph," IEEE Transactions on Wireless Communications, vol. 7, no. 2, pp. 574-583, February 2008.

[3] F.H.P. Fitzek, M. Dohler, I. Opperman, A. Molinaro, M. Katz, and C. Bettstetter (editors), Springer Journal on Wireless Personal Communications - Special Issue on Cooperation in Wireless Networks, vol. 43, no. 1, January 2007.

[4] S. Katti, S. Gollakotta, and D. Katabi, "Embracing wireless interference: Analog network coding," in Proc. ACM SIGCOMM, pp. 397-408, Kyoto, Japan, August 27-31, 2007.

[5] G. Kramer, I. Maric, and R.D. Yates, "Cooperative communications," Foundations and Trends in Networking, vol. 1, issue 3-4, Now Publishers, June 2007.

[6] G. Kramer, R. Berry, A. El Gamal, H. El Gamal, M. Franceschetti, M. Gastpar, and J.N. Laneman (editors), IEEE Transactions on Information Theory - Special Issue on Models, Theory, and Codes for Relaying and Cooperation in Communication Networks, vol. 53, no. 10, Oct. 2007.

[7] J.N. Laneman, D.N.C. Tse, and G.W. Wornell, "Cooperative diversity in wireless networks: Efficient protocols and outage behavior," IEEE Transactions on Information Theory, vol. 50, no. 12, pp. 3062-3080, Dec. 2004.

[8] L.E. Li, R. Alimi, D. Shen, H. Vishwanathan, and Y.R. Yang, "A general algorithm for interference alignment and cancellation in wireless networks," in Proc. IEEE INFOCOM, San Diego, CA March 15-19, 2010.

[9] C. Peng, Q. Zhang, M. Zhao, and Y. Yao, "On the performance analysis of network-coded cooperation in wireless networks," in Proc. IEEE INFOCOM, pp. 1460-1468, Anchorage, Alaska, May 6-12, 2007.

[10] S. Sharma, Y. Shi, J. Liu, and Y.T. Hou, "Is network coding always good for cooperative communciations," in Proc. IEEE INFOCOM, San Diego, CA, March 15-19, 2010.

[11] Y. Shi, S. Sharma, Y.T. Hou, and S. Kompella, "Optimal relay assignment for cooperative communications," in Proc. ACM MobiHoc, pp. 3-12, Hong Kong, China, May 27-30, 2008.

[12] Y. Tu and G.J. Pottie, "Coherent cooperative transmission from multiple adjacent antennas to a distant stationary antenna through AWGN channels," in Proc. IEEE Vehicular Technology Conference, pp. 130-134, Birmingham, AL, May 6-9, 2002.

[13] L. Xiao, T.E. Fuja, J. Kliewer, and D.J. Costello, "A network coding approach to cooperative diversity," IEEE Transactions on Information Theory, vol. 53, no. 10, pp. 3714-3722, Oct. 2007.

[14] H. Xu and B. Li, "XOR-assisted cooperative diversity in OFDMA wireless networks: Optimization framework and approximation algorithms," in Proc. IEEE INFOCOM, pp. 21412149, Rio de Janeiro, Brazil, April 19-25, 2009. 